5 Júlia Mayans, ${ }^{a}$ Mercè Font-Bardia ${ }^{b}$ and Albert Escuer ${ }^{*} a$

6

${ }^{\mathrm{a}}$ Departament de Química Inorgànica i Orgànica, Secció Inorgànica and Institute of Nanoscience

(IN2UB) and Nanotecnology, Universitat de Barcelona, Martí i Franquès 1-11, Barcelona-08028, Spain.

\title{
E-mail: albert.escuer@qi.ub.edu
}

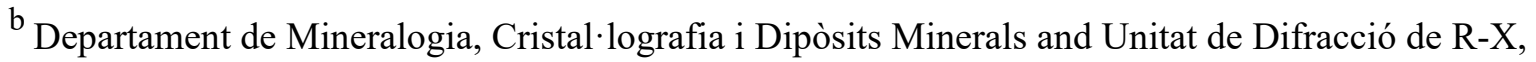


30 A wide family of clusters with the Na2MnIII 6 LnIII core derived from an enantiomerically pure or a

31 racemic mixture of Schiff bases lead to the characterization of a unique case of chiral/meso isomers with

$32 \mathrm{C} 3 / \mathrm{D} 3 \mathrm{~d}$ symmetry respectively, involving the rearrangement of the cluster bond parameters and the

33 change from hepta- to hexacoordination of the central LnIII cation. 
36 Supramolecular chemistry is defined as the chemistry of molecular assemblies and of the intermolecular

37 bond, 1 based on the key molecular recognition concept. Supramolecular chirality 2 is an emerging subfield

38 that focuses its attention on selfassembled entities built from chiral components. Employment of chiral

39 ligands in coordination chemistry induces the phenomenon of chirality transfer that produces clusters with

40 predetermined chirality at several hierarchical levels, from the ligand to the coordination sphere of the cations, to the molecule or the whole network.3,4

On one hand, the comparison between clusters synthesized from a chiral or racemic mixture of ligands is rarely performed because interest in these complexes is directly related to chirality and associated properties (stereoselective catalysis, sensing, chiroptical effects, etc.) and on the other hand, because it is generally assumed that the systems obtained from the racemic ligands will be a symmetric meso form or a simple mixture of both enantiomers, related by inversion centers or reflection planes in the network, maintaining the molecular core and very similar bond parameters. Nevertheless, this assumption is not a rule and in some cases radical differences have been found as was reported for the case of centrosymmetric meso [ $\mathrm{Ni2} 2 \mathrm{Fe} 2]$ or [Cu2 $\mathrm{Ln} 2]$ rings obtained from a racemic mixture of chiral organic ligands and (R)- or (S)-[Ni2Fe2]n or [Cu2Ln2]n chiral chains obtained from enantiomerically pure organic ligands.5,6

Noteworthily, in these cases the different dimensionality is based on how the building blocks are spatially arranged but always the inner structure and the connectivity between them are maintained. Interestingly, the differences between the meso and chiral forms in these examples go beyond structural and symmetric considerations and are also reflected in their magnetic response, because of the different dimensionality: single molecule magnet (SMM) behavior for discrete clusters and single chain magnet (SCM) response for 1D systems.5,6

Following our work on the properties of ligands involving chirality7-9 and selecting the (Rac)-, (R)- or (S)-H2L Schiff base obtained from the condensation of o-vanilline and the enantiomerically pure (S)-, (R)- or (Rac)-phenylglycinol precursor, Scheme 1, we report in this communication the characterization of nonanuclear clusters [Na2MnIII $6 \mathrm{TbIII}(\mathrm{Cl}) 6(\mathrm{~L}) 6(\mu 4-\mathrm{O}) 2](\mathrm{OH})$ (1Rac), (1R), (1S), [Na2MnIII 6 TbIII(Br)6(R-L)6( $\mu 4-\mathrm{O}) 2] \mathrm{Br}(2 \mathrm{~S})$ and [Na2MnIII $6 \mathrm{GdIII}(\mathrm{Cl}) 6(\mathrm{~L}) 6(\mu 4-\mathrm{O}) 2](\mathrm{OH})$ (3Rac) and $(3 \mathrm{~S})$ in which $\mathrm{L} 2-$ is the dianionic form of $\mathrm{H} 2 \mathrm{~L}$ (synthetic details in ESI $\dagger$ ).

The reported complexes provide unprecedented and unexpected examples of the relationship between chiral and meso isomerism, which, among others, involves changes in the coordination number of the lanthanide cation and does not fit with the conventional definitions of isomerism.

The centrosymmetric complex 1Rac can be envisaged as two heterometallic [NaMnIII 3 TbIII] trigonal bipyramidal subunits sharing the TbIII cation which is placed in one of the apical sites, resulting in nonanuclear [NaI 2MnIII 6 TbIII] cluster with D3d symmetry, Fig. 1, and ESI, Tables S1 and S3. $\uparrow$ The L2-ligand links the lanthanide, manganese and sodium cations and the inversion center present on the 
cations are related by a $\mathrm{C} 3$ axis and thus become equivalent inside each subunit. One oxo donor is placed in the center of the equilateral triangular arrangement of MnIII cations with $\mathrm{Mn1}-\mathrm{O}-\mathrm{Mn1}$ ' bond angles very close to $120^{\circ}$.

The MnIII cations are coordinated by the oxo donor, the iminic nitrogen from one L2- ligand, one O-alkoxo donor that links each manganese atom with the lanthanide ion, one O-phenoxo atom that bridges with the sodium cation and two $\mu-\mathrm{Cl}$ bridges with the neighboring MnIII cations, resulting in an octahedral coordination with the Jahn-Teller elongation axis directed towards the trans-chloro donors. The coordination polyhedron around the hexacoordinated TbIII ion consists of an octahedron compressed along the $\mathrm{C} 3$ axis, with an $\mathrm{O} 1-\mathrm{Tb}-\mathrm{O} 1^{\prime}$ bond angle of 97.65(9) ${ }^{\circ}$, Fig. 2 and ESI, Table S3. $\dagger$ All Mn1-O1$\mathrm{Tb}$ bond angles are identical, $117.5(1)^{\circ}$, and the TbIII cation is equidistant to $\mathrm{O} 4$ and $\mathrm{O}^{\prime}$ with a noncontact distance of $2.988 \AA$. Each subunit shows a propeller shape (helicate) that turns in opposite sense and, consequently, the NaI cations show the opposite $\Delta / \Lambda$ configuration. Complex $3 \mathrm{Rac}$ is isostructural to 1 Rac with practically identical structural parameters (ESI, Tables S1 and S3†).

The structure of the 1R complex is similar to $1 \mathrm{Rac}$ in its general aspects but the lack of inversion center reduces its symmetry to $\mathrm{C} 3$. The main consequences of this fact are related to the coordination environment around the TbIII cation that becomes heptacoordinated with six shorter $\mathrm{Tb}-\mathrm{O}$ bond distances to the six O-alkoxo donors (Tb-O1 2.310(6) $\AA$, Tb-O4 2.282(6) $\AA$ ) and one larger Tb-O8 distance of $2.688(11) \AA$ to one of the $\mu 4$-oxo ligands, resulting in one apicated octahedron, Fig. 2. This distance is shorter than the $\mathrm{Tb} \cdots \mathrm{O} 7$ distance of $3.281 \AA$, showing the clear displacement of the TbIII cation from the centre of the complex towards the $\mu 4-\mathrm{O}$ oxo donor O8, resulting in an asymmetrization of $0.593 \AA$.

The core bond parameters show several distortions to facilitate the coordination of the $\mu 4-\mathrm{O}$ donor to the TbIII cation: the $\mathrm{O} 4-\mathrm{Tb}-\mathrm{O} 44^{\prime}$ bond angle increases up to $103.2(2)^{\circ}$, larger than $\mathrm{O} 1-\mathrm{Tb}-\mathrm{O} 1^{\prime}$ which reduces to $91.0(2)^{\circ}$, the $\mathrm{Mn} 2-\mathrm{O} 4-\mathrm{Tb}$ bond angle becomes shorter than $\mathrm{Mn} 1-\mathrm{O} 1-\mathrm{Tb}\left(111.0(3)^{\circ}\right.$ vs. 124.0 $\left.(3)^{\circ}\right)$ and the $\mathrm{O} 8$ oxo donor is displaced $0.187 \AA$ out of the plane defined by the three $\operatorname{Mn}(2)$ cations towards the lanthanide cation, Fig. 2, whereas on the other side of the molecule, $\mathrm{O} 7$ is placed $0.076 \AA$ out of the plane defined by the three Mn cations towards the $\mathrm{Na}(1)$ cation (Na1-O8 2.8110 $\AA$ ) and can be

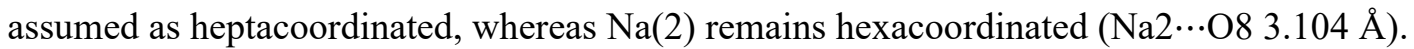

Complexes 1R, 1S, 2S and 3S, in which we have modified selectively the L2- enantiomer ( $\mathrm{R}$ or $\mathrm{S})$, the LnIII cation (TbIII or GdIII) or the axial co-ligand ( $\mathrm{Cl}-$ or $\left.\mathrm{Br}^{-}\right)$, show a similar core, confirming that these features are common to a large series of complexes (ESI, Tables S2 and S4 $\dagger$ ).

The complexes obtained from the racemic mixtures could yield three kinds of products: the meso form in which the different conformations of the ligands are related by Sn symmetry elements placed in the molecule, a pair of enantiopure isomers in the same crystal related by symmetry in the network or more rarely, the enantiomeric resolution in separate crystals. Among them, experimental structural data show that the meso form is the preferred option for 1Rac and 3Rac. On the other hand, reproducing the synthesis with a mixture containing an enantiomeric excess of $(\mathrm{S})-\mathrm{H} 2 \mathrm{~L}$ providing adequate stoichiometry 
ESI $\dagger$ ), showing that, in this case, the meso isomer is preferred to the chiral cluster. This feature could be related to the relative rotation of both subunits which minimizes the repulsion between them for the meso form, Fig. 1. Noteworthily, previously reported related systems obtained from achiral ligands have also been shown to be centrosymmetric. 10

Modification of the substituents on the ligands is a usual synthetic tool to obtain different products on the basis of the involved packing forces, solubility or intermolecular interactions. From this point of view, the reported examples of meso/chiral isomerism could be assumed as a subtle but similar effect. However, the features observed for the [Na2MnIII 6 LnIII] family of clusters are completely new because the packing of the meso or chiral clusters is identical even at the level of the torsion angles of the pendant phenyl groups (ESI, Fig. S2 $\uparrow$ ) and the only difference is the symmetry centre (R3 vs. R3- space group) and thus, the phenomenon appears to be strictly intramolecular and symmetry dependent. From the hostguest point of view the size of the central cation is a determinant: an analogous chiral [NaMnIII $6 \mathrm{MnII}$ ] system with one smaller MnII cation instead of the LnIII ion exhibits an MnIIO6 octahedral environment,7,8 whereas the lanthanides are large enough to establish contact with the seventh O-donor at distances of around $2.7 \AA$. It becomes crucial to note that this arrangement is the consequence of the possibility of being centrosymmetric but not due to the alternative arrangement in the network of $\mathrm{S}$ and $\mathrm{R}$ clusters related by inversion centres and that retaining the most usual heptacoordination of the LnIII cation could be equally possible (or even more probable a priori), in light of the structures of the chiral clusters and the flexibility of L2-.

O-centred MnIII 3 triangles with axial bridging chloro ligands are scarce and have been revealed to show typical ferromagnetic coupling.7,11,12 Susceptibility measurements in the 2-300 K range of temperatures were performed for the reported complexes. In all cases the $\chi \mathrm{MT}$ plots show a continuous increase from room temperature up to a maximum of $\chi \mathrm{MT}$ around $\sim 20 \mathrm{~K}$ followed by a strong decrease at low temperature. The room temperature values agree with the expected ones for six $\mathrm{S}=2$ and one TbIII cation $(29.8 \mathrm{~cm} 3 \mathrm{~mol}-1 \mathrm{~K})$ or one GdIII cation $(25.6 \mathrm{~cm} 3 \mathrm{~mol}-1 \mathrm{~K})$ with g values slightly lower than 2.00 .

Isotropic fit of the GdIII complexes up to the $\chi \mathrm{MT}$ maximum with the two-J Hamiltonian derived from the scheme shown in the Fig. 3 inset yields the best values $\mathrm{J} 1=+2.3 \mathrm{~cm}-1, \mathrm{~J} 2=-0.11 \mathrm{~cm}-1$ and $\mathrm{g}$ $=1.89$ for $3 \mathrm{Rac}$ and $\mathrm{J} 1=+3.4 \mathrm{~cm}-1 \mathrm{~J} 2=-0.20 \mathrm{~cm}-1$ and $\mathrm{g}=1.89$ for $3 \mathrm{~S}$ (PHI program, 13 2J convention).

As can be expected from the structural information the chiral and meso clusters exhibit a similar but not identical magnetic response. The fit confirms the ferromagnetic interaction inside the triangular MnIII 3 fragments and suggests weak antiferromagnetic interactions between the ferromagnetic MnIII 3 units and the central lanthanide. However, the strong decay at lower temperatures indicates a moderately strong anisotropy that is evidenced in the magnetization measurements, reaching values clearly lower than that expected for an isotropic system, (ESI $\dagger$ ). The TbIII complexes follow a similar pattern to that of the GdIII ones. 
148 In conclusion, a unique case of strictly intramolecular meso/chiral isomerism has been found, in which,

149 in addition to the conventional supramolecular recognition tools such as the size and spatial distribution

150 of O-donors, also the symmetry is involved. The most interesting feature of the reported systems is that

151 for the very first time we report a symmetry-dependent case of intramolecular meso-chiral isomerism that

152 does not fit with the conventional nomenclature (the same shape and dimensionality but different

153 connectivity), involving the full rearrangement of the bond parameters and the change in the coordination

154 numbers of the cations. The employment of symmetry elements to modify the dimensionality or the

155 coordination sphere of the cations is an emerging supramolecular tool that is demonstrated to be useful

156 for the rational design of unusual isomers with coordination environments such as LnIII cations or d-

157 cations with tunable coordination spheres. In a further paper the complete series of [Na2MnIII 6 LnIII]

158 complexes from CeIII to YbIII central cations will be explored to determine the size effect of the cation

159 along the f-block.

160 
161 ACKNOWLEDGEMENTS

162

163 This work was supported by ministerio de ciencia, innovación y universidades (spain), project pgc2018164 094031-b-100.

165 


\section{REFERENCES}

1681 J. M. Lehn, Supramolecular Chemistry, VCH-Weinheim, 1995.

1692 M. Liu, L. Zhang and T. Wang, Chem. Rev., 2015, 115, 7304.

1703 H. Miyake, Symmetry, 2014, 6, 880.

1714 J. Crassous, Chem. Soc. Rev., 2009, 38, 830.

1725 N. Hoshino, Y. Sekine, M. Nihei and H. Oshio, Chem. Commun., 2010, 46, 6117.

1736 T. Ueno, T. Fujinami, N. Matsumoto, M. Furusawa, R. Irie, N. Re, T. Kanetomo, T. Ishida and Y. 174 Sunatsuki, Inorg. Chem., 2017, 56, 1679.

1757 A. Escuer, J. Mayans, M. Font-Bardia, M. Górecki and L. Di Bari, Dalton Trans., 2017, 46, 6514.

1768 J. Mayans, M. Font-Bardia, L. Di Bari and A. Escuer, Chem. - Eur. J., 2018, 24, 18705.

1779 J. Mayans, M. Font-Bardia and A. Escuer, Inorg. Chem., 2018, 57, 926.

17810 P.-P. Yang, X.-L. Wang, L.-C. Li and D.-Z. Liao, Dalton Trans., 2011, 40, 4155.

17911 C. Ding, C. Gao, S. Ng, B. Wang and Y. Xie, Chem. - Eur. J., 2013, 19, 9961.

18012 P.-P. Yang, L.-L. Zhu, Y. Xu and C.-Y. Shao, Z. Anorg. Allg. Chem., 2013, 639, 1821.

18113 N. F. Chilton, R. P. Anderson, L. D. Turner, A. Soncini and K. S. Murray, J. Comput. Chem., $182 \quad 2013,34,1164$. 


\section{Legends to figures}

186 Scheme 1 Coordination to one MnIII, one LnIII and one Na cations for

187 the L2- ligand employed in this work. The asterisk denotes the chiral

188 carbon.

190 Figure. 1 Top, view of the molecular structure of 1Rac. Down, axial view of

191 1Rac (left) and 1R (right) showing the rotation between subunits and the

192 distances between centroids. Color code: MnIII, dark green; GdIII, light

193 blue; $\mathrm{Na}$, orange; $\mathrm{N}$, navy; $\mathrm{O}$ red; $\mathrm{Cl}$, violet.

195 Figure 2. Top, labelled view of the common core of clusters 1R, 1S, 2S and

196 3S. Middle, labelled common core of complexes 1Rac and 3Rac. Down,

197 Coordination environment of the chiral and meso isomers of complexes

$198 \quad 1-3$.

199

200 Figure 3. Plot of $\chi \mathrm{MT}$ product vs. T for 1R (diamonds), $2 \mathrm{~S}$ (triangles), 3Rac

201 (blue) and 3S (red). Inset, the coupling scheme for compounds 3. Solid

202 lines show the best fit of the experimental data in the $20-300 \mathrm{~K}$ range

203 of temperatures.

204

205

206 


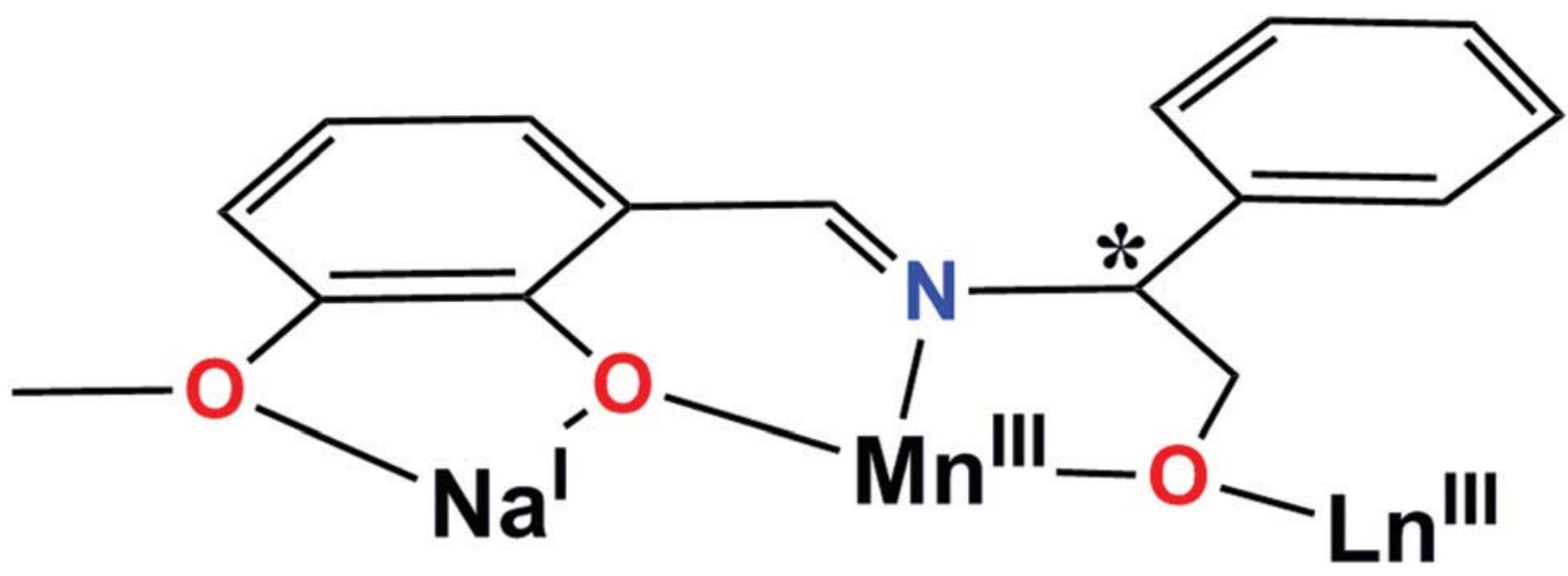


FIGURE 1
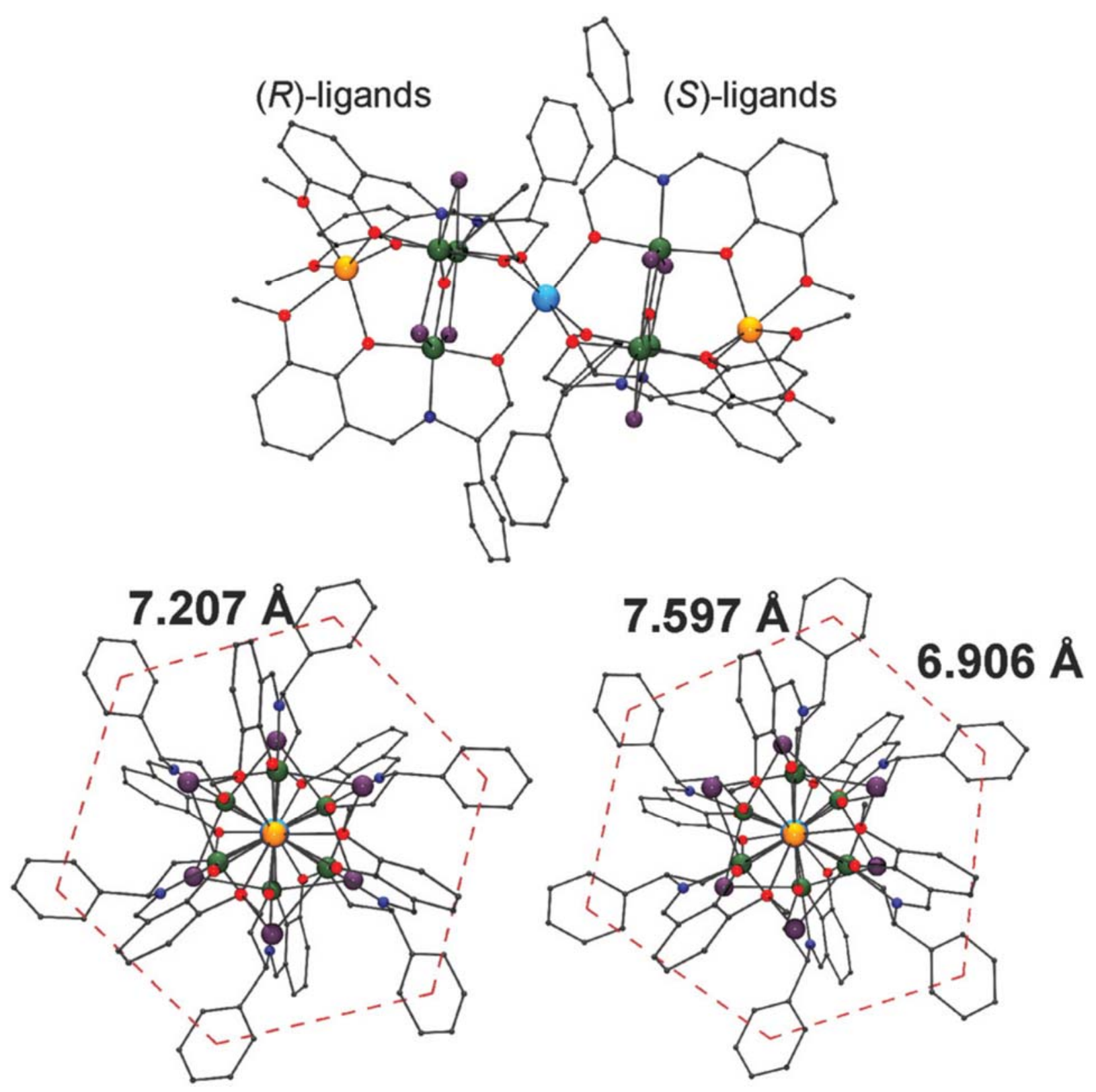
FIGURE 2
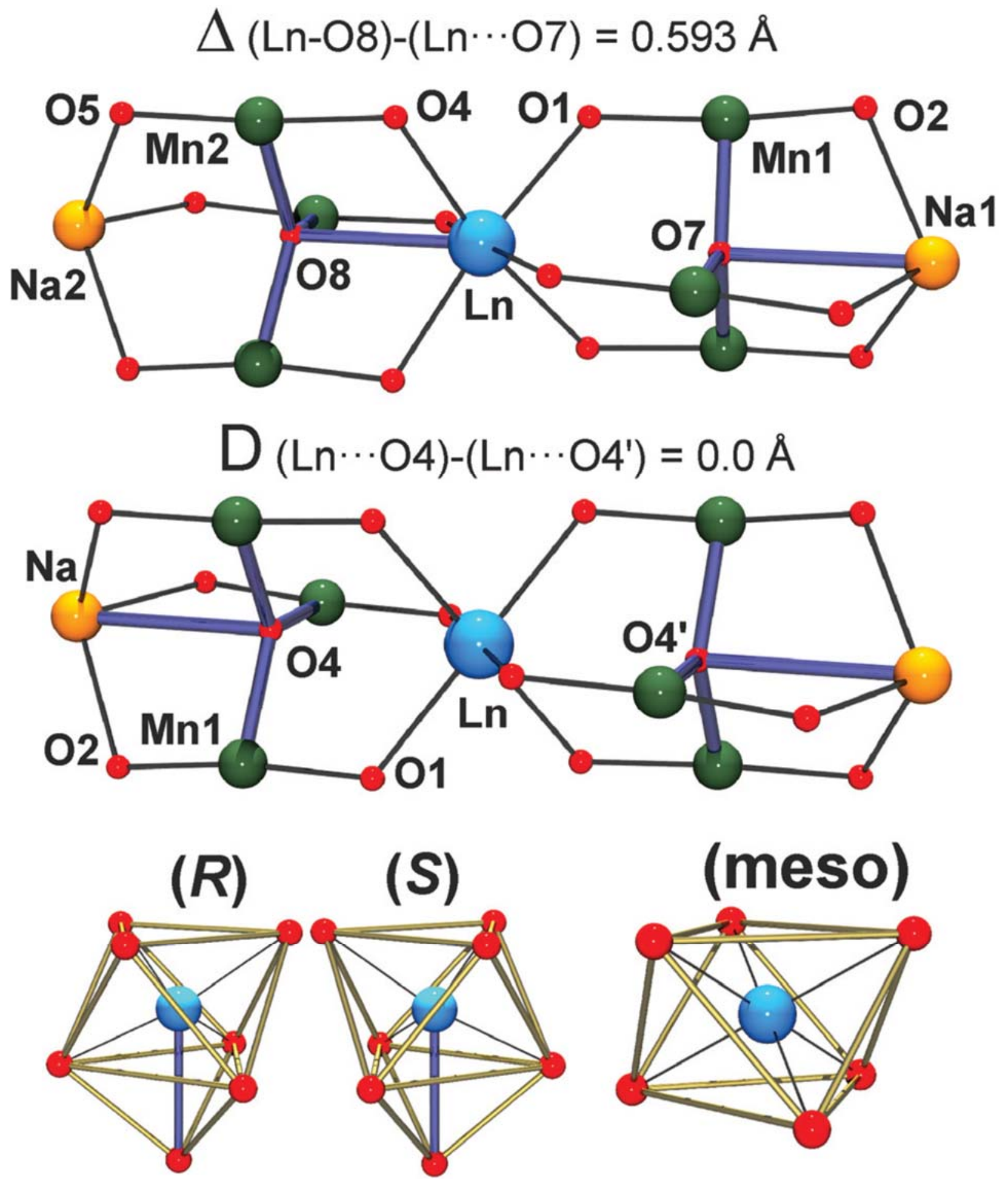
FIGURE 3

318

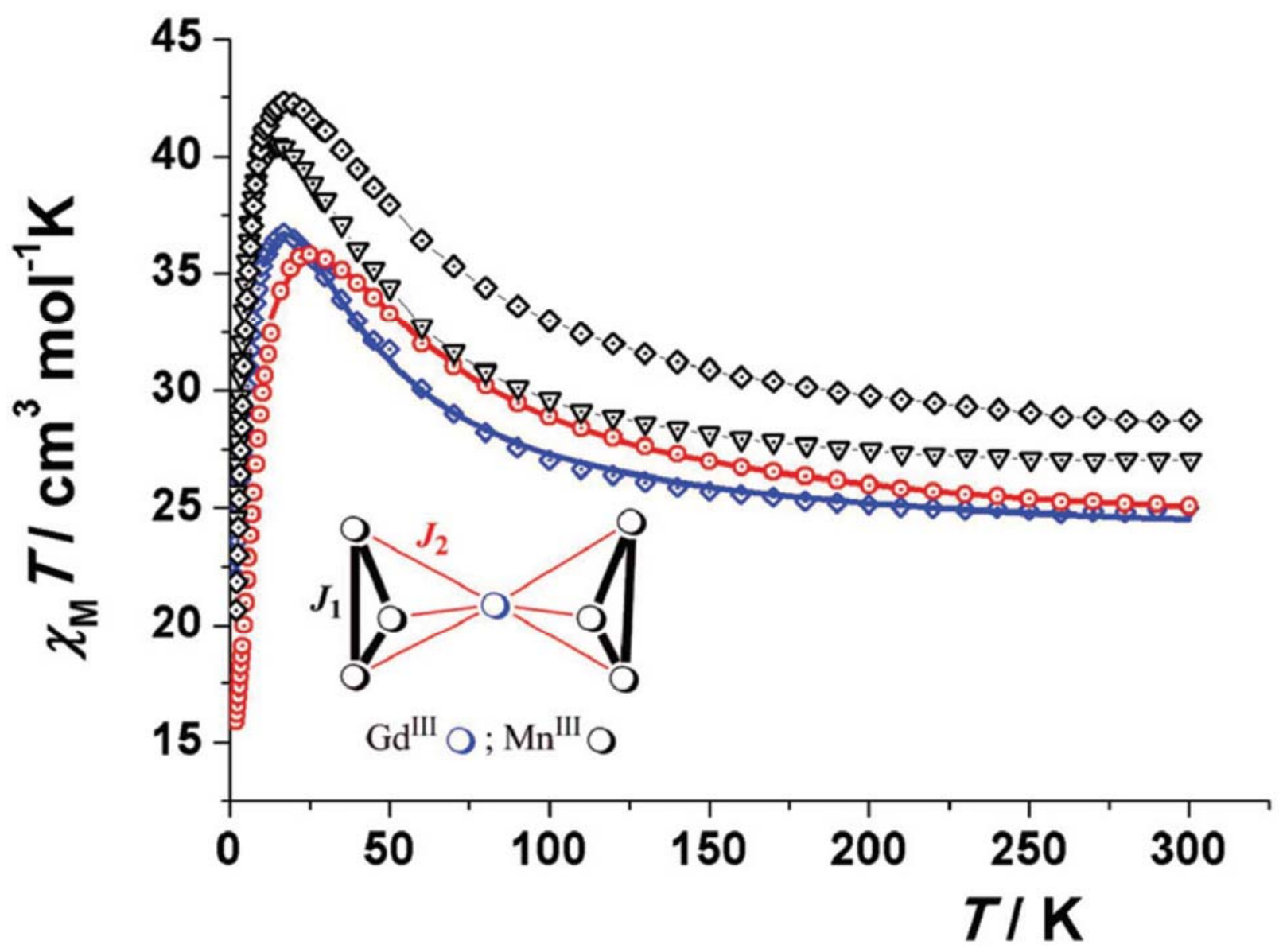

